\title{
Corrigendum
}

\section{Corrigendum to "Predictors of Recovery from Traumatic Brain Injury-Induced Prolonged Consciousness Disorder"}

\author{
Hiroaki Abe $\mathbb{D}^{1,2}$ Keigo Shimoji, ${ }^{3}$ Yoshihide Nagamine, ${ }^{4}$ Satoru Fujiwara, ${ }^{5}$ \\ and Shin-Ichi Izumi ${ }^{2,6}$ \\ ${ }^{1}$ Department of Rehabilitation Medicine, Kohnan Hospital, Tohoku Ryogo Center, Sendai, Japan \\ ${ }^{2}$ Department of Physical Medicine and Rehabilitation, Graduate School of Medicine, Tohoku University, Sendai, Japan \\ ${ }^{3}$ Department of Diagnostic Radiology, Tokyo Metropolitan Geriatric Hospital, Tokyo, Japan \\ ${ }^{4}$ Department of Neurosurgery, Kohnan Hospital, Tohoku Ryogo Center, Sendai, Japan \\ ${ }^{5}$ Department of Neurosurgery, Kohnan Hospital, Sendai, Japan \\ ${ }^{6}$ Graduate School of Biomedical Engineering, Tohoku University, Sendai, Japan
}

Correspondence should be addressed to Hiroaki Abe; abehi0827@gmail.com

Received 27 October 2020; Accepted 27 October 2020; Published 9 November 2020

Copyright (C) 2020 Hiroaki Abe et al. This is an open access article distributed under the Creative Commons Attribution License, which permits unrestricted use, distribution, and reproduction in any medium, provided the original work is properly cited.

In the article titled "Predictors of Recovery from Traumatic Brain Injury-Induced Prolonged Consciousness Disorder" [1], one subscore of the Kohnan score was omitted from the appendix in error. The correct Kohnan score should compose of 7 subscores, and the corrected appendix is as follows: 
TABLe 7

\begin{tabular}{|c|c|c|c|c|c|}
\hline Clinical symptoms & $\begin{array}{l}\text { Grade } \\
\text { Extreme (10) }\end{array}$ & Severe (9) & Moderate (8/7) & Mild (5) & Slight $(0)$ \\
\hline $\begin{array}{l}\text { Voluntary } \\
\text { movement }\end{array}$ & $\begin{array}{l}\text { (1) Absent } \\
\text { (2) Acrocontracture } \\
\text { (3) Pain reflex but } \\
\text { slight trembling } \\
\text { and rough } \\
\text { breathing }\end{array}$ & $\begin{array}{l}\text { (1) Almost absent } \\
\text { but parts of the } \\
\text { extremities move } \\
\text { minutely } \\
\text { (2) Part of the } \\
\text { extremity flexed } \\
\text { and part } \\
\text { paralyzed } \\
\text { (3) Pain reflex or no } \\
\text { pain reflex with } \\
\text { clearly frowning } \\
\text { face }\end{array}$ & $\begin{array}{l}\text { (1) Occasional } \\
\text { all/partial } \\
\text { extremity } \\
\text { movement with no } \\
\text { intention } \\
\text { (2) Extremity could be } \\
\text { paretic } \\
\text { (3) Brushing away } \\
\text { reaction for pain }\end{array}$ & $\begin{array}{l}\text { (1) Occasional } \\
\text { movement to meet } \\
\text { an object } \\
\text { (2) Capable of raising the } \\
\text { arms upward or } \\
\text { moving them in the } \\
\text { intended direction, } \\
\text { that is, face or head, } \\
\text { imitating a posture of } \\
\text { the tester }\end{array}$ & $\begin{array}{l}\text { (1) Capable of } \\
\text { movement with } \\
\text { intention } \\
\text { (2) Capable of } \\
\text { unassisted } \\
\text { posture change } \\
\text { (partial change } \\
\text { inclusive) } \\
\text { (3) Moving } \\
\text { wheelchair } \\
\text { unassisted, even } \\
\text { if awkwardly }\end{array}$ \\
\hline $\begin{array}{l}\text { Voluntary } \\
\text { ingestion }\end{array}$ & $\begin{array}{l}\text { Totally incapable of } \\
\text { masticating and } \\
\text { swallowing; on tube } \\
\text { nutrition } \\
\text { (gastric/nasal } \\
\text { feeding) }\end{array}$ & $\begin{array}{l}\text { (1) Almost on tube } \\
\text { nutrition } \\
\text { (2) Saliva } \\
\text { swallowing or } \\
\text { mastication is } \\
\text { found } \\
\text { (3) Capable of } \\
\text { attempting slight } \\
\text { perusal } \\
\text { ingestion, that is, } \\
\text { fruit juice, } \\
\text { custard pudding, } \\
\text { and so forth }\end{array}$ & $\begin{array}{l}\text { (1) Capable of } \\
\text { masticating; even if } \\
\text { not, almost capable } \\
\text { of assisted peroral } \\
\text { ingestion by } \\
\text { swallowing, though } \\
\text { sometimes choking } \\
\text { (2) Insufficient peroral } \\
\text { ingestion requires } \\
\text { tube nutrition }\end{array}$ & $\begin{array}{l}\text { (1) Capable of unassisted } \\
\text { ingestion by } \\
\text { swallowing; } \\
\text { mastication could be } \\
\text { awkward } \\
\text { (2) Capable of ingesting } \\
\text { all the rice gruel } \\
\text { served or chopped } \\
\text { food with assistance } \\
\text { (3) Attempting to reach } \\
\text { the mouth with a } \\
\text { passed spoon or put } \\
\text { the food into the } \\
\text { mouth awkwardly }\end{array}$ & $\begin{array}{l}\text { Ingesting on own } \\
\text { using spoon } \\
\text { awkwardly }\end{array}$ \\
\hline $\begin{array}{l}\text { Fecal and urinary } \\
\text { incontinence }\end{array}$ & $\begin{array}{l}\text { No observed somatic } \\
\text { movement when } \\
\text { evacuating/urinating }\end{array}$ & $\begin{array}{l}\text { Slight somatic } \\
\text { movement when } \\
\text { evacuating/urinating }\end{array}$ & $\begin{array}{l}\text { After incontinence, a } \\
\text { displeased look or } \\
\text { some signal is } \\
\text { observed, that is, } \\
\text { frequent somatic } \\
\text { movement }\end{array}$ & $\begin{array}{l}\text { (1) Forced regular } \\
\text { evacuating and } \\
\text { urinating leads to the } \\
\text { prevention of fecal } \\
\text { and urinary } \\
\text { incontinence } \\
\text { (2) Communicating the } \\
\text { fact in a certain way } \\
\text { after incontinence }\end{array}$ & $\begin{array}{l}\text { Except during the } \\
\text { night, preevacuation } \\
\text { and preurination } \\
\text { communication is } \\
\text { possible }\end{array}$ \\
\hline $\begin{array}{l}\text { Ophthalmography } \\
\text { and visual } \\
\text { recognition }\end{array}$ & $\begin{array}{l}\text { (1) Eyes not opened } \\
\text { (2) Eyes opened, no } \\
\text { blink reflex }\end{array}$ & $\begin{array}{l}\text { (1) Eyes opened, } \\
\text { blink reflex } \\
\text { (2) No following } \\
\text { ocular } \\
\text { movement, and } \\
\text { no focusing eyes } \\
\text { on an object }\end{array}$ & $\begin{array}{l}\text { (1) Looking straight } \\
\text { toward the } \\
\text { direction of the call } \\
\text { (2) Following a moving } \\
\text { object or staring at } \\
\text { a TV, although } \\
\text { understanding is } \\
\text { impossible }\end{array}$ & $\begin{array}{l}\text { (1) Discriminating close } \\
\text { relatives followed by } \\
\text { a facial expression } \\
\text { (2) Favorite picture, } \\
\text { among other things, } \\
\text { induce a facial } \\
\text { expression }\end{array}$ & $\begin{array}{l}\text { (1) Capable of } \\
\text { reading easy } \\
\text { words } \\
\text { (2) Capable of } \\
\text { understanding } \\
\text { simple numbers } \\
\text { (3) When watching } \\
\text { TV, response and } \\
\text { laughter are } \\
\text { apparent }\end{array}$ \\
\hline $\begin{array}{l}\text { Vocalizing and } \\
\text { utterances }\end{array}$ & $\begin{array}{l}\text { (1) No vocalizing } \\
\text { (2) No lip } \\
\text { movement under } \\
\text { tracheostomy }\end{array}$ & $\begin{array}{l}\text { (1) Groaning etc., } \\
\text { without } \\
\text { meaningful } \\
\text { utterances } \\
\text { (2) Lip movement } \\
\text { observed under } \\
\text { tracheostomy }\end{array}$ & $\begin{array}{l}\text { (1) A short utterance } \\
\text { though not } \\
\text { understandable } \\
\text { (2) Occasional } \\
\text { inarticulate vocal } \\
\text { response to calls } \\
\text { (3) Under } \\
\text { tracheostomy, } \\
\text { response to calls is } \\
\text { through lip } \\
\text { movement }\end{array}$ & $\begin{array}{l}\text { (1) Occasional vocalizing } \\
\text { of a meaningful word } \\
\text { (2) Vocal response to } \\
\text { calls } \\
\text { (3) Imitating talking by } \\
\text { the tester under } \\
\text { tracheostomy }\end{array}$ & $\begin{array}{l}\text { (1) Capable of } \\
\text { vocalizing a } \\
\text { simple word } \\
\text { response } \\
\text { (2) Lip movement } \\
\text { corresponds to } \\
\text { what is asked }\end{array}$ \\
\hline
\end{tabular}


TABLe 7: Continued.

\begin{tabular}{|c|c|c|c|c|c|}
\hline Clinical symptoms & $\begin{array}{l}\text { Grade } \\
\text { Extreme (10) }\end{array}$ & Severe (9) & Moderate $(8 / 7)$ & Mild (5) & Slight $(0)$ \\
\hline $\begin{array}{l}\text { Response and } \\
\text { comprehension }\end{array}$ & No response to calls & $\begin{array}{l}\text { Some response to } \\
\text { calls, such as somatic } \\
\text { or eye movement, } \\
\text { etc. }\end{array}$ & $\begin{array}{l}\text { Response to calls is } \\
\text { possible at times, but } \\
\text { no understanding }\end{array}$ & $\begin{array}{l}\text { Response and } \\
\text { understanding of simple } \\
\text { calls is possible at times }\end{array}$ & $\begin{array}{l}\text { Response fits the } \\
\text { purpose of calls and } \\
\text { nearly correct } \\
\text { understanding }\end{array}$ \\
\hline $\begin{array}{l}\text { Change of } \\
\text { expression }\end{array}$ & $\begin{array}{l}\text { No response to } \\
\text { ambient sound } \\
\text { stimulations and TV } \\
\text { sounds, etc. }\end{array}$ & $\begin{array}{l}\text { Change of } \\
\text { expression, such as } \\
\text { smiling, crying, and } \\
\text { anger, is not due to } \\
\text { ambient } \\
\text { stimulations }\end{array}$ & $\begin{array}{l}\text { Change of expression } \\
\text { is occasionally found in } \\
\text { response to ambient } \\
\text { stimulations }\end{array}$ & $\begin{array}{l}\text { Change of expression, } \\
\text { such as smiling, crying } \\
\text { and anger closely } \\
\text { matches an expected } \\
\text { response to the ambient } \\
\text { stimulation }\end{array}$ & $\begin{array}{l}\text { Change of } \\
\text { expression, such as } \\
\text { crying and smiling, } \\
\text { exactly matches an } \\
\text { expected response to } \\
\text { the ambient } \\
\text { stimulation }\end{array}$ \\
\hline
\end{tabular}

\section{References}

[1] H. Abe, K. Shimoji, Y. Nagamine, S. Fujiwara, and S.-I. Izumi, "Predictors of recovery from traumatic brain injury-induced prolonged consciousness disorder," Neural Plasticity, vol. 2017, Article ID 9358092, 11 pages, 2017. 